\section{Resistant TB: use the tools available}

Rwanda's experience in combating multi-drug-resistant tuberculosis (MDR-TB) offers lessons in using available tools wisely now, rather than waiting for the results of long-term scientific investment (Nature 493, 14-16; 2013).

Of 306 patients who began standardized 'second-line' drug regimens for MDR-TB through a Rwandan programme in 2005-09, 270 (88.2\%) were successfully treated. Nearly half of the patients were HIV-positive and all lived in extreme poverty.

Crucially, Rwanda's Ministry of Health provided each patient with close follow-up care and with monthly support towards nutrition and transport. As a result, only six patients (2\%) defaulted on their treatment.

Such common-sense interventions prevent disease progression and facilitate directly observed therapy. But they are seldom included in costeffectiveness studies - much less in nationwide strategies.

Once drug-supply chains and comprehensive support for adherence to treatment are in place, such positive MDR-TB treatment outcomes will be possible all over rural Africa. Agnes Binagwaho* Ministry of Health, Kigali, Rwanda. agnes_binagwaho@hms.harvard. edu

${ }^{*}$ On behalf of 5 co-signatories (see go.nature.com/ttsddo for full list).

\section{Resistant TB: remove cultural barriers}

Social and cultural obstacles confront practitioners trying to control the spread of multi-drugresistant tuberculosis (TB; Nature 493, 14-16; 2013). Removing these barriers will improve adherence to drug regimes and impede the development of drug resistance.

For example, young Asian women with TB may not come forward for treatment because they see it as a threat to their marriage prospects. Alcohol consumption, which is prevalent among some Caucasian patients, can interfere with drug treatments. And recovering patients who return to their jobs, often away from home, may drop contact with health-care providers.

Besides supplying medicines and efficient health-service management, it is vital to promote culturally adjusted messages in particular, to emphasize why patients with TB must stick to their lengthy treatments.

While waiting for shorter and less-toxic drug regimens to become available on a large scale, non-governmental organizations such as Médecins Sans Frontières include socio-cultural support to help promote adherence. This strategy is founded on medical anthropology - the applied study of traditional beliefs and practices relating to illness and health care. Such support helps to ensure effective treatment and follow-up (see, for example, go.nature. com/4b6f9f).

Gilles Guerrier Noumea, New Caledonia. guerriergilles@gmail.com

\section{Altmetrics: research council responds}

Heather Piwowar lists several funders, including the UK Medical Research Council (MRC), that "still explicitly ask for a list of research papers rather than products" in their grantapplication process (Nature 493, 159; 2013). However, we at the MRC strongly agree that other outputs can provide valuable evidence of research significance and impact.

These research 'products' include development of new interventions, production and distribution of widely used data sets, changes to clinical practice or other advances that influence policy decisions, and commercial indicators such as licence income and growth of spin-out companies.

The MRC considers all such products in assessing researcher track records. Our grant-

application forms specifically ask researchers how they intend to manage and share the results of their work, and to outline their productivity beyond published papers.

In addition, the company Researchfish has developed a structured online system for MRC-funded scientists to record their publications and researchproduct output. The system also provides publicly accessible information relating to MRC grants. It has now been adopted by 15 other UK funding agencies. Ian Viney Medical Research Council, London. ian.viney@headoffice.mrc.ac.uk

\section{Altmetrics: too soon for use in assessment}

I question the worth of many of the altmetrics ('alternative metrics') that Heather Piwowar discusses for evaluating a researcher's total output

(Nature 493, 159; 2013).

Altmetrics include, for example, the number of downloads, 'likes' or shares for a research-related YouTube video, tweet or blog post. Although these may provide insight into how such research 'products' have influenced the community and the public, they lack authority and credibility as a performance measure, not least because it is easy to cheat by creating multiple accounts.

Grant reviewers cannot therefore rely on such data as indicators of the value of their associated research products. In fact, the US National Science Foundation's new funding policy, around which Piwowar bases her arguments, requires the research products to be in citable and accessible form - implying that it is only the number of citations in scientific journals that will be taken into account in assessing the value of these products.

I believe that it is premature to integrate most altmetrics, apart from citation statistics, into research-assessment schemes at a time when the merits of publication quantity versus quality are still being debated (N. Haslam and S. M. Laham Eur. J. Soc. Psychol. 40, 216-220; 2010).

Man Kit Cheung The Chinese University of Hong Kong, Hong Kong.

mkcheung@cuhk.edu.hk

\section{Aristocratic names get short shrift}

In his discussion of suspects in the Piltdown Man mystery, Chris Stringer alludes to the French Jesuit priest, philosopher and palaeontologist Pierre Teilhard de Chardin (Nature 492, 177-179; 2012). The article mistakenly shortens the great man's compound surname to "de Chardin". In fact, it should read "Teilhard de Chardin" or just "Teilhard".

Such aristocratic last names are often misinterpreted in English. One glaring example is the condition known as Tourette's syndrome, incompletely named after Georges Gilles de la Tourette, who first described it. "Gilles" is part of the compound last name and not a middle name, as is commonly assumed.

Antoine Louchart Ecole Normale Supérieure de Lyon, Lyons, France.

antoine.louchart@ens-lyon.fr

\section{CORRECTION}

A table in the Outlook article 'Nanotechnology: Carrying drugs' (Nature 491 (suppl. 7425), S58-S60; 2012) wrongly stated that Cerulean Pharma's drug CRLX101 is in phase I trials. In fact, it has been in phase II trials since mid2012. The table also incorrectly gave the company name as "Cerulean Pharmaceuticals". 